\title{
EFFECTS OF DECEPTIVE SELF-REPORTS OF QUITTING ON THE RESULTS OF TREATMENT TRIALS FOR SMOKING: A QUANTITATIVE ASSESSMENT
}

\author{
Joel L. Weissfeld, ${ }^{1,2 *}$ JAMes J. Holloway ${ }^{1,2}$ and John P. KIRSChT ${ }^{3}$ \\ 'Medical Service, Veterans Administration Medical Center, Ann Arbor, Michigan, ${ }^{2}$ Department \\ of Internal Medicine, University of Michigan, Ann Arbor, Michigan and ${ }^{3}$ Department of Health \\ Behavior and Health Education, University of Michigan, Ann Arbor, Michigan, U.S.A.
}

(Received in revised form 8 August 1988)

\begin{abstract}
Problems with self-report measures for smoking motivate the use of biochemical tests in treatment trials for smoking. These biochemical tests, unfortunately, are not perfect. In this paper, we present an algebraic model of bias in treatment trials for smoking. Bias is expressed in terms of the deception rate among continued smokers in a control group, the relative deception rate among continued smokers in an experimental group, and the sensitivity and specificity of a biochemical test which may be used either to confirm self-reports of quitting or to replace self-report entirely. For given test specificity and sensitivity, the model defines deception rates for which different biochemical testing strategies are preferred. The model is presented in the context of current knowledge on the phenomenon of deception among adult smokers. The paper concludes that better judgements regarding the role of biochemical tests in treatment trials for smoking require more precise information regarding the magnitude and determinants of deception.
\end{abstract}

Smoking Truth disclosure Models theoretical Research design

\section{INTRODUCTION}

The methods of the randomized, controlled, clinical trial have been employed to determine whether varied treatments help smokers quit. A self-report of quitting represents the most direct measure of the outcome of such treatments. However, studies suggest that a smoker may not accurately report his true smoking behavior [1-19]. Further, a smoker who has knowingly received a special, intensive, or otherwise experimental treatment for smoking may be more inclined to misrepresent his smoking [20]. Within the context of a clinical trial, where unobtrusive or blinded smoking cessation interventions are difficult to implement, the greatest

*All correspondence should be addressed to: Joel L. Weisefeld, M.D., M.P.H., Ambulatory Care Section, Veterans Administration Medical Centre, 2215 Fuller Road, Ann Arbor, MI 48105, U.S.A.

Support provided by the Veterans Administration, Health Services Research and Development. concern is that biased self-reports may produce an apparent treatment effect where none, in fact, exists [21].

This concern forms the basis for the use of biochemical measures as an outcome for clinical trials which examine treatments for smoking [22]. Usually, biochemical tests are used to confirm the reports of those who say they have quit. Unlike self-report, the veracity of biochemical tests are independent of subjects' knowledge of the treatments delivered. Any test, however, will misclassify some quitters and continued smokers. Investigators will generally accept misclassification of some quitters, as a consequence of testing strategies which correctly identify all or most smokers. In the context of a clinical trial, this is a conservative strategy; as we will show, it is designed to prevent biased self-report from giving the false appearance of treatments with different effects. We will also show that, when subjects are relatively truthful in their self-reports, a testing strategy which 
favors identification of continued smoking will more severely underestimate any real difference among treatments.

This paper presents a model which quantifies bias in the results of a clinical trial. Bias results from deception by continued smokers in the experimental and control groups and from imperfections in biochemical tests used to detect deception. From this model, we derive equations which express the conditions under which different uses of biochemical tests appear more or less appropriate.

\section{BACKGROUND}

\section{Sensitivity and specificity of biochemical tests for smoking}

None of the current biochemical measures for smoking is perfect. Concentrations of carbon monoxide in exhaled air, carboxyhemoglobin in blood, and thiocyanate, nicotine, and cotinine in blood, saliva, and urine, distinguish smokers and nonsmokers. Assays for these substances vary with respect to sensitivity, specificity, cost, ease of automation, and suitability for large scale field studies. Using populations presumed to have "no reason" to misrepresent smoking status, previous studies $[3,10,11,23-25]$ of the validity of these measures compare either one measure against a second or one measure against self-report. Thus, a test, validated against self-report in one setting, is often used to detect deceptive self-reports in another setting. This contributes to the uncertainty which surrounds quantification of deceptive selfreport.

All the biochemical measures lose sensitivity at lower levels of smoking [23, 24]. Sensitivity is defined as the proportion of true smokers who are classified as smokers by the biochemical test. In addition, the substances assayed have different biological half-lives [22]. These biochemical measures are more or less susceptible to losses in sensitivity which result from longer durations since last cigarette (which correlate, in turn, with lower levels of smoking) [25]. Smokers who participate in studies designed to assess the sensitivity of biochemical tests may differ in important ways from smokers who participate in clinical trials. These differences may be exaggerated among clinical trial participants who falsely report quitting, the subgroup for which biochemical tests are generally intended. For example, smokers in an experimental group or smokers who falsely report quitting may, on average, smoke less heavily, abstain for longer durations before a scheduled collection of biological samples for assay, inhale less deeply, or switch to cigarettes with lower nicotine yields.

Biochemical measures of smoking are more or less susceptible to losses in specificity which result from a variety of environmental exposures [26, 27]. Specificity is defined as the proportion of true nonsmokers who are classified as nonsmokers by the biochemical test. All measures are susceptible to errors which result from failure to account for pipe, cigar, and passive smoking. Assessments of exhaled carbon monoxide and blood carboxyhemoglobin are particularly susceptible to exposures from occupational sources, air pollution, or faulty home heating. Assessments of thiocyanate are susceptible to variation in dietary intakes of cyanide and thiocyanate. Assessments of nicotine and cotinine must account for usage of smokeless tobacco and nicotine containing chewing gum. Of particular interest are exsmokers who require medical care for a chronic condition related to smoking or who quit smoking as a result of an experimental intervention. They may differ from the groups of nonsmokers typically used to establish the specificity of biochemical measures of smoking. Such exsmokers, for example, may have greater exposures to passive smoke, environmental sources of carbon monoxide, or even dietary sources of thiocyanate. Each of these exposures potentially lowers the specificity of a biochemical test.

Few studies assess all the customary biochemical measures of smoking on a single group of subjects. The study by Jarvis et al. [6] is an exception. This study assessed all measures on 211 patients attending a cardiology or peripheral vascular disease clinic. Twenty-one professed nonsmokers, with very high plasma cotinine levels, were reclassified as smokers. When subjects were subsequently classified by cutoffs which yielded $95 \%$ specificity for each assay, sensitivities, for detection of all smokers, ranged between $25 \%$ (for saliva thiocyanate) and $98 \%$ (for urinary cotinine). Thus, different biochemical measures are imperfect and differ with respect to sensitivity (for given specificity).

We argue that comparison of biochemical tests can involve criteria in addition to sensitivity and specificity. Tests with differences in sensitivity and specificity (and associated differences in cost or ease of administration) can be evaluated in the context of the magnitude of 
deception which threatens the validity of clinical trials for smoking.

\section{Deception}

Several factors, in theory, influence deception rates. The pressure to under-report smoking derives from the social undesirability of smoking, from threats to self-confidence or self-image which result from failure to quit smoking, or from wishes to please counselors or experimenters with whom smokers engage. In some studies, participants are intentionally made aware of plans to use a biochemical assay to validate self-reports, in the hope that this socalled "bogus pipeline" improves the accuracy of self-report [28]. Hypothetically, the bogus pipeline can be imagined to work in "reverse". For example, some nonsmokers, made aware that self-reports of nonsmoking are subject to biochemical confirmation, may be prompted to report, falsely, that they smoke. Such a tendency might result from concern over the inconvenience associated with the collection of the biological sample or from the fear that biological fluid, so collected, might be used for some unspecified, unapproved, or undesired purpose (e.g. illicit drug screening or AIDS testing). Finally, apparent deception among continued smokers who report not smoking may not represent deception, as such, but rather inadequacy of the survey measure used to assess smoking status [29]. The point, here, is that a number of factors are capable of influencing the proportion of smokers who appear to falsely report not smoking. Moreover, many of these factors are amenable to control by investigators.

With this discussion as background, Tables 1-3 summarize previous investigations which enable an estimate of deception rates. Table 1 summarizes five studies which examine either general population samples, individuals who consent to health screening examinations, or members of an occupational group [1-5]. Estimates for the deception rate, the proportion of smokers who deny smoking, range between 0.6 and $7.8 \%$. Some of this variation probably derives from variation in biochemical measures and associated cutoffs used to identify deceptive self-reports among professed nonsmokers. In general, these studies employ test combinations and cut-off values expected to produce relatively specific measures for smoking. Thus, the deception rates suggested by the studies cited in Table 1 cannot be simply attributed to falsely positive test results in a largely nonsmoking

Table 1. Deception rates inferred from community surveys, occupational surveys, or health screening studies

\begin{tabular}{|c|c|c|c|c|}
\hline Source & Biochemical measure & $\begin{array}{l}\text { Cutoff used to } \\
\text { distinguish smoker } \\
\text { from nonsmoker }\end{array}$ & Sample size* & $\begin{array}{l}\text { Deception } \\
\text { rate }(d)\end{array}$ \\
\hline Fortmann et al. (1984) [1] & $\begin{array}{l}\text { Exhaled carbon monoxide } \\
\text { and serum thiocyanate }\end{array}$ & $\begin{array}{c}8 \mathrm{ppm} \\
100 \mu \mathrm{mol} / 1\end{array}$ & 380 & 0.0316 \\
\hline Pierce et al. (1987) [2] & Saliva cotinine & $44 \mathrm{ng} / \mathrm{ml}$ & 622 & 0.0402 \\
\hline Wald et al. (1981) [3] & Serum carboxyhemoglobin & $3 \%$ & 2095 & 0.0060 \\
\hline Cohen and Bartsch (1980) [4] & $\begin{array}{l}\text { Serum thiocyanate } \\
\text { Carboxyhemoglobin }\end{array}$ & $150 \mu \mathrm{mol} / 1$ & $\begin{array}{l}438 \\
462\end{array}$ & $\begin{array}{l}0.0274 \\
0.0779\end{array}$ \\
\hline Robertson et al. (1987) [5] & $\begin{array}{l}\text { Carboxyhemoglobin } \\
\text { Serum thiocyanate } \\
\text { and exhaled carbon monoxide }\end{array}$ & $\begin{array}{c}3 \% \\
70 \mu \mathrm{mol} / 1 \\
10 \mathrm{ppm}\end{array}$ & $\begin{array}{r}443 \\
54\end{array}$ & $\begin{array}{l}0.0384 \\
0.0556 \dagger\end{array}$ \\
\hline
\end{tabular}

-Self-reported quitters with a positive biochemical test for smoking and all self-reported smokers.

fIncludes two self-reported nonsmokers who confess smoking when challenged with elevated thiocyanate results.

Table 2. Deception rates inferred from patients selected from clinical populations

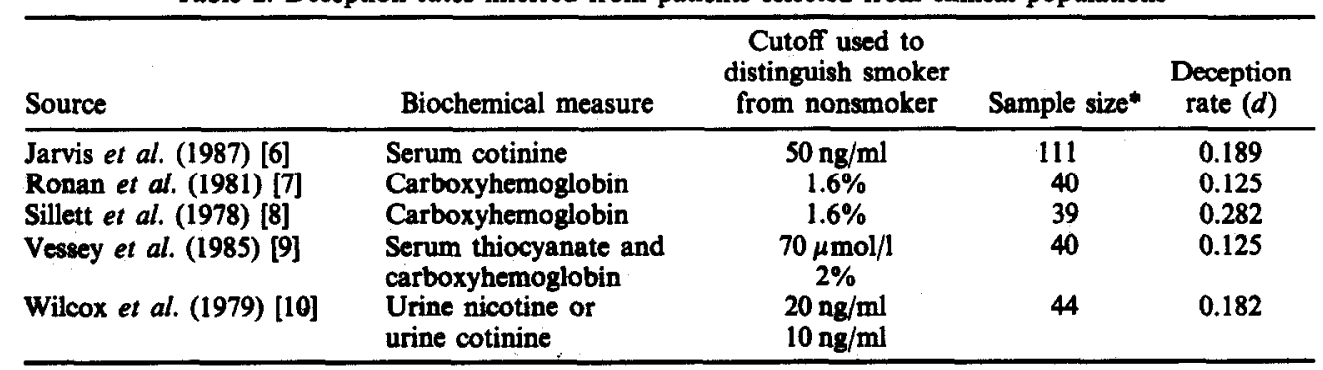

*Self-reported quitters with a positive biochemical test for smoking and all self-reported smokers. 
Table 3. Deception rates inferred from subjects enrolled in stop smoking clinics or smoking cessation treatment trials.

\begin{tabular}{|c|c|c|c|c|}
\hline Source & Biochemical measure & $\begin{array}{l}\text { Cutoff used to } \\
\text { distinguish smoker } \\
\text { from nonsmoker }\end{array}$ & Sample size* & $\begin{array}{l}\text { Deception } \\
\text { rate }(d)\end{array}$ \\
\hline Abrams et al. (1987) [11] & Saliva cotinine & $10 \mathrm{ng} / \mathrm{ml}$ & $\begin{array}{r}81 \\
4\end{array}$ & $\begin{array}{l}0.0123 \\
0\end{array}$ \\
\hline $\begin{array}{l}\text { Colletti et al. (1982) [12] } \\
\text { (four separate studies) }\end{array}$ & Exhaled carbon monoxide & $10 \mathrm{ppm}$ & $\begin{array}{l}15 \\
14 \\
28\end{array}$ & $\begin{array}{l}0.1333 \\
0 \\
0\end{array}$ \\
\hline Glynn et al. (1986) [13] & Exhaled carbon monoxide & $10 \mathrm{ppm}$ & 36 & 0.194 \\
\hline Jamrozik et al. (1984) [14] & Urinary cotinine & $100 \mathrm{ng} / \mathrm{ml}$ & 51 & 0.216 \\
\hline Lando, (1975) & Exhaled carbon monoxide & $11 \mathrm{ppm}$ & 8 & 0 \\
\hline Ohlin et al. (1976) [15] & Carboxyhemoglobin & $0.8 \%$ & 209 & 0.167 \\
\hline Russell et al. (1976) [16] & Carboxyhemoglobin & $3.7 \%$ & 20 & 0.050 \\
\hline Sillett et al. (1978) [17] & Carboxyhemoglobin & $1.6 \%$ & 91 & 0.363 \\
\hline Stookey et al. (1987) [18] & $\begin{array}{l}\text { Saliva cotinine } \\
\text { Exhaled carbon monoxide }\end{array}$ & $\begin{array}{l}10 \mathrm{ng} / \mathrm{ml} \\
8 \mathrm{ppm}\end{array}$ & $\begin{array}{l}262 \\
262\end{array}$ & $\begin{array}{l}0.149 \\
0.084\end{array}$ \\
\hline Walker and Franzini (1985) [19] & Exhaled carbon monoxide & $8 \mathrm{ppm}$ & 49 & 0 \\
\hline
\end{tabular}

*Self-reported quitters with a positive biochemical test for smoking and all self-reported smokers.

population (i.e. individuals who report not smoking).

Table 2 summarizes five studies which enable calculation of a deception rate among smokers examined in the context of a physician visit for illnesses associated with cigarette smoking [6-10]. Deception rates range between 12.5 and $28.2 \%$. Though some employ slightly less specific measures for smoking, these studies clearly indicate the potential for deception among this select group of smokers.

Table 3 summarizes 10 studies which enable calculation of deception rates among subjects completing defined treatments for smoking [11-19]. Calculated deception rates range between 0 and $36.3 \%$ (weighted average $14.9 \%$, sample size 868 ). Only one study specifically delivered treatments in the context of the care provided by physicians [14]. These studies examine treatments which vary widely in intensity, duration, nature of subject-experimenter contacts, and conditions under which self-reports are obtained.

In summary, available data suggest low deception rates (less than $8 \%$ ) among participants of community survey or health screening exams, high rates (approximately $20 \%$ ) among patients visiting doctors' offices, and widely varying rates among subjects completing an experimental treatment for smoking. Unfortunately, very few studies compare deception rates between groups of smokers who complete experimental and control treatments for smoking. The Multiple Risk Factor Intervention Trial (MRFIT) did employ a relatively nonspecific measure of smoking, blood thiocyanate, to adjust selfreported cigarette smoking quit rates, in both experimental and control groups [20]. Deception rates were estimated from observed and expected serum thiocyanate levels, the latter adjusted for diuretic, pipe, and cigar use. Deception rates in the control (Usual Care) group ranged between 1.2 and $3.0 \%$ (depending on duration of follow-up). Corresponding deception rates in the experimental (Special Intervention) group ranged between 8.8 and $17.2 \%$. Estimated deception rates in the Special Intervention group may be inflated. The regression methods used to calculate expected thiocyanate levels disregard the fact that cigarette smokers who switch to cigars or pipes (which may have occurred at a higher rate in the Special Intervention group) have higher thiocyanate levels than cigar or pipe smokers who have never smoked cigarettes [30]. Special Intervention participants, as well, received concomitant dietary treatments aimed at reducing serum cholesterol. The effects of induced dietary changes on levels of thiocyanate are uncertain. However, the results of MRFIT provide, at least, an upper bound on the expected enhancement in deception among smokers who receive an experimental treatment for smoking.

\section{DESCRIPTION OF THE MODEL}

\section{Definitions and assumptions}

We derive equations which express the bias in a clinical trial produced by deception among continued smokers, by enhanced deception among continued smokers who receive an experimental treatment, and by errors in a biochemical test administered to none, some, or all 
participants in the trial. For this purpose, we define

$P_{1}$, the true quit rate in the experimental group (i.e. the proportion of subjects in the experimental group who truly quit smoking);

$P_{0}$, the true quit rate in the control group (i.e. the proportion of subjects in the control group who truely quit smoking);

$d$, the deception rate among continued smokers in the control group (i.e. the proportion of continued smokers in the control group who falsely report quitting);

$f$, a factor which represents enhanced deception by continued smokers in the experimental group;

$f \cdot d$, the proportion of continued smokers in the experimental group who falsely report quitting;

$S_{\mathrm{p}}$, the specificity of the biochemical test for smoking (i.e. the proportion of true nonsmokers identified as nonsmokers by the biochemical assay;

$S_{\mathrm{e}}$, the sensitivity of the biochemical test for smoking (i.e. the proportion of true smokers identified as smokers by the biochemical assay).

We assume that the sensitivity and specificity of the test are independent of both treatment category and self-reported smoking status. Further, we assume that the experimental treatment is at least as effective as the control treatment (i.e. $\left.P_{1} \geqslant P_{0}\right)$. Finally, we assume that all subjects, who truly quit smoking, report that they have quit.

The true treatment effect is the difference between the quit rates in the experimental and control groups (i.e. $P_{1}-P_{0}$ ). The absolute bias is the difference between the observed treatment effect and the true treatment effect. Finally, the relative bias is the ratio between the bias and the true treatment effect.

\section{PROPERTIES OF THE MODEL}

\section{Basic relationships}

An algebraic model is used to derive expressions for bias when trial outcomes are defined by self-report alone, biochemical test results alone, and a combination of self-report and biochemical test results. We derive these expressions in Appendix A. The relative bias, under conditions of self-report, is

$$
\frac{d \cdot\left(f\left(1-P_{1}\right)-\left(1-P_{0}\right)\right)}{P_{1}-P_{0}} \text {. }
$$

When the biochemical test is used to classify all participants, the relative bias is

$$
-\left(\left(1-S_{\mathrm{p}}\right)+\left(1-S_{\mathrm{e}}\right)\right) \text {. }
$$

Finally, when the test is only used to classify self-reported quitters, the relative bias is

$$
d \cdot\left(f\left(1-P_{1}\right)-\left(1-P_{0}\right)\right) \frac{1-S_{e}}{P_{1}-P_{0}}-\left(1-S_{\mathrm{p}}\right) .
$$

Several generalizations result directly from these expressions.

Case I: $\boldsymbol{P}_{1}=\boldsymbol{P}_{0}=\boldsymbol{P}$. In the absence of a true treatment effect (i.e. $P_{1}=P_{0}=P$ ), one can examine the absolute, but not the relative, bias. Expressions for the absolute bias are found in Appendix A (expressions A4, A9, and A12). When $P_{1}=P_{0}=P$, the absolute bias, in a trial which classifies subjects by self-report, is

$$
d(1-P)(f-1) . \quad(4, \text { from A4) }
$$

From expression (A9), we conclude that a trial is not biased when a test, no matter how imperfect, is used to classify all subjects. The absolute bias, in a trial which classifies only self-reported quitters according to test results, is

$$
d(1-P)(f-1)\left(1-S_{\mathrm{e}}\right) . \quad(5, \text { from A12) }
$$

Therefore, in the absence of a treatment effect, the study is not biased if a biochemical test classifies all subjects (expression A9). (Unless deception rates are identical among experimental and control subjects, $f=1$, expression 5), administration of a biochemical test only to self-reported quitters always biases the trial in favor of the experimental treatment. In this latter case, the degree to which the assay reduces the bias (relative to self-report alone) depends only on the sensitivity of the assay. Finally, we note that in the absence of a true treatment effect, use of a test with imperfect specificity does not bias the results of the trial.

Case II: $d=0 ; P_{1}>P_{0}$. In the absence of deception (i.e. $d=0$ ), it follows from expression (1) that a trial which relies solely on self-report is not biased.

However, a trial which classifies all participants according to an imperfect test (i.e. $S_{\mathrm{e}}<1$ and or $S_{\mathrm{p}}<1$ ) has a relative bias which is the negative of the sum of the "false positive" $\left(1-S_{p}\right)$ and "false negative" $\left(1-S_{e}\right)$ rates 
(expression 2). A negative relative bias reduces the apparent magnitude of a true treatment effect. Here, we assume that the sum of the sensitivity and specificity is greater than one. This implies that the test discriminates between smokers and nonsmokers in the direction intended by those who make use of the test.

When the test is used to classify self-reported quitters only, a relative bias exists only if the test has imperfect specificity. Here, the relative bias is the negative of the "false positive" rate, i.e. $-\left(1-S_{\mathrm{p}}\right)$ (from expression 3 ).

Case III: $f=1 ; P_{1}>P_{0}$. In the absence of enhanced deception among continued smokers who receive an experimental intervention $(f=1)$, expression (1), the relative bias when the test is not used, becomes

$$
-d
$$

the negative of the deception rate among smokers in the control group. Expression (2), the relative bias when testing all subjects, does not change. Expression (3), the relative bias associated with testing self-reported quitters alone, reduces to

$$
-\left(d\left(1-S_{\mathrm{e}}\right)+\left(1-S_{\mathrm{p}}\right)\right) .
$$

Thus, under these conditions (Case III), the relative bias, is always negative. That is, whether the test is administered to none, all subjects, or self-reported quitters only, a negative bias reduces the apparent magnitude of a true treatment effect. These three settings vary only with respect to the magnitude of this negative relative bias. The relative bias is less (in absolute value) when the test is administered to all participants (relative to no testing at all) when

$$
\begin{aligned}
& \mid \text { expression (2)|<|expression (6)|, } \\
& \left|-\left(\left(1-S_{\mathrm{p}}\right)+\left(1-S_{\mathrm{e}}\right)\right)\right|<|-d|,
\end{aligned}
$$

or

$$
\left(1-S_{\mathrm{p}}\right)+\left(1-S_{\mathrm{e}}\right)<d,
$$

(i.e. when the sum of the "false positive" and the "false negative" rates is less than the deception rate). The relative bias is less (in absolute value) when the test is administered to only selfreported quitters (relative to no testing at all) when

$$
\begin{aligned}
& \mid \text { expression (7) }|<| \text { expression (6) } \mid, \\
& \left|-\left(d\left(1-S_{e}\right)+\left(1-S_{\mathrm{p}}\right)\right)\right|<|-d|,
\end{aligned}
$$

or

$$
\frac{1-S_{\mathrm{p}}}{S_{\mathrm{e}}}<d,
$$

(i.e. when the ratio between the "false positive" rate and the sensitivity ("true positive" rate) is less than the deception rate). Finally, the relative bias is always less when self-reported quitters, instead of all participants, are tested (i.e. $\left|-d\left(\left(1-S_{\mathrm{e}}\right)+\left(1-S_{\mathrm{p}}\right)\right)\right|$ is always less than $\left.\left|-\left(\left(1-S_{\mathrm{e}}\right)+\left(1-S_{\mathrm{p}}\right)\right)\right|\right)$. Thus, when deception is equivalent among experimental and control groups (i.e. $f=1$ ), a trial which classifies subjects by the results of an imperfect test remains biased in a direction against the true treatment effect. The false positive and false negative rates (when all subjects are tested; expression 8) and the false positive rate (when only self-reported quitters are tested; expression 9) must be small relative to the deception rate if testing is to diminish the relative bias which exists by virtue of deception alone.

Summary. These special cases are easily summarized (see Table 4). In the presence of

\begin{tabular}{|c|c|c|c|c|c|}
\hline \multirow[b]{2}{*}{ Case } & \multicolumn{2}{|r|}{ Conditions } & \multicolumn{3}{|c|}{ Outcome assessment } \\
\hline & $\begin{array}{l}\text { Treatment } \\
\text { effects }\end{array}$ & Deception & Self-report & $\begin{array}{l}\text { Test self-reported } \\
\text { quitters }\end{array}$ & $\begin{array}{l}\text { Test all } \\
\text { subjects }\end{array}$ \\
\hline I & $\begin{array}{c}\text { Equal } \\
\left(P_{1}=P_{0}\right)\end{array}$ & $\begin{array}{l}\text { Present, enhanced } \\
\quad(d>0, f>1)\end{array}$ & + & + & 0 \\
\hline II & $\begin{array}{l}\text { Unequal } \\
\left(P_{1}>P_{0}\right)\end{array}$ & $\begin{array}{l}\text { Absent } \\
(d=0)\end{array}$ & 0 & - & - \\
\hline III & $\begin{array}{l}\text { Unequal } \\
\left(P_{1}>P_{0}\right)\end{array}$ & $\begin{array}{c}\text { Present, not enhanced } \\
(d>0, f=1)\end{array}$ & - & - & - \\
\hline $\begin{array}{l}\text { General } \\
\text { case }\end{array}$ & $\begin{array}{l}\text { Unequal } \\
\left(P_{1}>P_{0}\right)\end{array}$ & $\begin{array}{l}\text { Present, enhanced } \\
\quad(d>0, f>1)\end{array}$ & + & $\pm^{*}$ & - \\
\hline
\end{tabular}
equivalent treatments (Case I), deception and enhanced deception produce a bias, unless all subjects are classified by the results of a biochemical test. The trial is biased in favor of the

Table 4. Expected direction of bias in the results of a clinical treatment trial for smoking. Results are shown for three alternative means of determining outcome and under four modeled conditions

$0=$ no bias; $+=$ positive bias; $-=$ negative bias.

*Depends on the ratio between the false positive and false negative rates (expression 10). 
experimental treatment. Testing self-reported quitters lessens the magnitude of the bias by a factor equal to the false negative rate $\left(1-S_{e}\right)$. In the absence of deception (Case II), a trial which uses an imperfect test to confirm selfreported smoking status is biased against observation of a treatment effect. The bias is larger if the test is used to classify all subjects (relative to self-reported quitters only). Finally, the absence of enhanced deception among continued smokers who receive the experimental intervention (Case III: $f=1$ ), a trial is always biased against observation of the treatment effect. Whether a confirmatory test increases or decreases (relative to no testing) the magnitude of the bias depends on the relationship between test characteristics (i.e. $S_{\mathrm{p}}, S_{\mathrm{c}}$ ) and the deception rate $(d)$. Again, the bias is larger when the test is used to classify all subjects (relative to self-reported quitters alone).

\section{The general case}

We now consider the general case, treatments with different effects $\left(P_{1}-P_{0}>0\right)$, deceptive self-report $(0<d \leqslant 1)$, and enhanced deception within the experimental group $(f>1$; see Table 4, general case). When outcomes are based solely on self-report, the trial is biased in favor of the experimental treatment (expression 1). When outcomes are based solely on biochemical test results, the trial is biased against the experimental treatment (expression 2). In addition, the sensitivity and specificity appear to have equivalent effects on the magnitude of the relative bias.

When the biochemical test is used only to classify self-reported quitters, the trial can be biased in either direction, depending on the relative magnitudes of the false negative $\left(1-S_{\mathrm{e}}\right)$ and false positive $\left(1-S_{\mathrm{p}}\right)$ rates (expression 3 ). Here, deterioration in sensitivity produces progressive bias in a positive direction. Deterioration in specificity has an effect in the opposite direction. Rearranging expression (3), set to values less than zero, we conclude that the relative bias is not positive as long as

$$
\frac{1-S_{\mathrm{p}}}{1-S_{\mathrm{e}}}>\frac{d}{P_{1}-P_{0}}\left(f\left(1-P_{1}\right)-\left(1-P_{0}\right)\right) \text {. }
$$

That is, this is the condition under which deception will not, at least, exaggerate the apparent effectiveness of the experimental treatment. Some representative values for $d, f, P_{1}$, and $P_{0}$ illustrate the properties of this inequality. Suppose $P_{1}=0.2, P_{0}=0.1, d=0.1$, and $f=2$.
Under these conditions, a clinical trial which tests self-reported quitters is unbiased if $\left(1-S_{\mathrm{p}}\right) /\left(1-S_{\mathrm{e}}\right)=0.7$, biased in a negative direction if $\left(1-S_{\mathrm{p}}\right) /\left(1-S_{\mathrm{e}}\right)>0.7$, and biased in a positive direction if $\left(1-S_{p}\right) /\left(1-S_{e}\right)<0.7$. Suppose the biochemical test for smoking misclassifies $10 \%$ of true nonsmokers as smokers (i.e. $1-S_{\mathrm{p}}=0.1$ ). Then, this test need only achieve a sensitivity of 0.857 (i.e. $1-0.1 / 0.7$ ) in order to guarantee a trial not biased in a positive direction.

The demands on the sensitivity of the test increase as $d$ and/or $f$ increases. Suppose the deception rate is 0.3 instead of 0.1 . Here, a trial which tests self-reported quitters is unbiased if $\left(1-S_{\mathrm{p}}\right) /\left(1-S_{\mathrm{e}}\right)=2.1$ and is negatively biased if $\left(1-S_{\mathrm{p}}\right) /\left(1-S_{\mathrm{e}}\right)>2.1$. When $S_{\mathrm{p}}=0.9$, the same value used above, $S_{\mathrm{e}}$ must exceed 0.952 to guarantee a trial not positively biased.

Thus, when sensitivity or specificity are not perfect, we can find the point beyond which improvement in test sensitivity (for a given test specificity) results in progressive bias in a negative direction.

\section{The effect of deception rates on the relative bias}

A clinical trial estimates, quantitatively, the degree to which an experimental treatment for smoking enhances smoking cessation. In the presence of biased self-report and/or imperfect biochemical confirmatory tests for smoking, this estimate, in general, is biased. Depending on the magnitude of deception $(d)$ and enhanced deception in the experimental group $(f)$, classification of treatment outcomes according to the results of a biochemical test may or may not decrease this bias. Again, depending on the values for $d$ and $f$, alternative testing strategies (e.g. testing only self-reported quitters vs testing all subjects) may affect bias differently.

In Figs 1-3, we compare, pairwise, three strategies for classification of outcomes: (1) self-reports only (strategy A), (2) biochemical test results only (strategy B), and (3) self-reports with biochemical testing restricted to selfreported quitters (strategy C). These three strategies form three paired comparisons. In Fig. 1, a trial which uses self-report is compared with a trial which uses biochemical testing uniformly. In Fig. 2, a trial which uses self-report is compared with a trial which confirms the reports of self-reported quitters. In Fig. 3, a trial which uses biochemical testing uniformly is compared with a trial which confirms the reports of self-reported quitters. 
Self-report (A) vs universal biochemical testing (B)

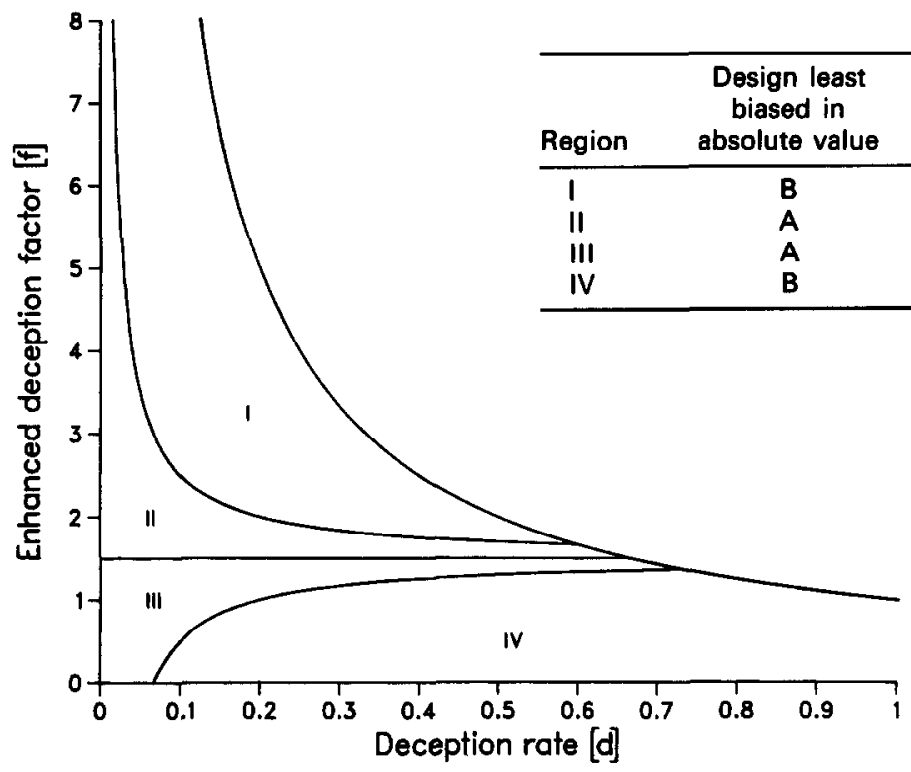

Fig. 1. Effect of deception rates on the expected relative bias. The figure shows four regions defining values for $d$ and $f$. Each region is unique with respect to the relative magnitudes (in absolute value) and direction of biases produced by two designs: outcomes classified solely by self-report (design A) and solely by biochemical test results (design $\mathrm{B}$ ). The figure shows modeled results for $P_{1}=0.4, P_{0}=0.1, S_{\mathrm{e}}=0.9$, and $S_{\mathrm{p}}=0.9$.

Self-report (A) vs selective biochemical testing (C)

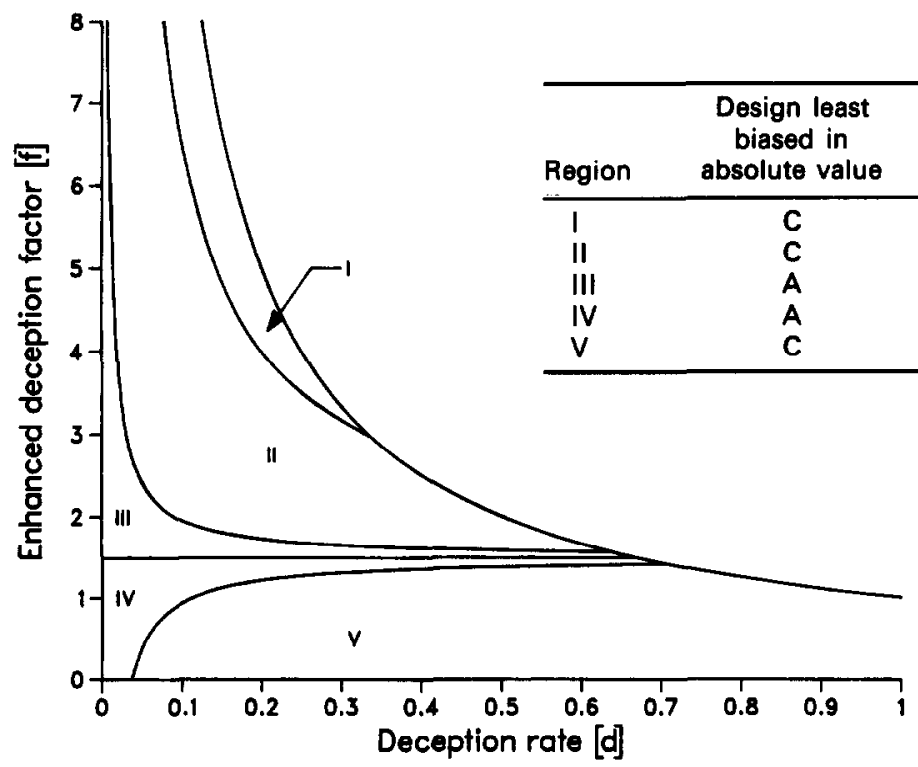

Fig. 2. Effect of deception rates on the expected relative bias. The figure shows five regions defining values for $d$ and $f$. Each region is unique with respect to the relative magnitudes (in absolute value) and direction of biases produced by two designs: outcomes classified solely by self-report (design A) and by a combination of self-report among self-reported smokers and of biochemical test results among selfreported quitters (design $C$ ). The figure shows modeled results for $P_{1}=0.4, P_{0}=0.1, S_{c}=0.9$, and $S_{\mathrm{p}}=0.9$. 
Universal (B) vs selective biochemical testing (C)

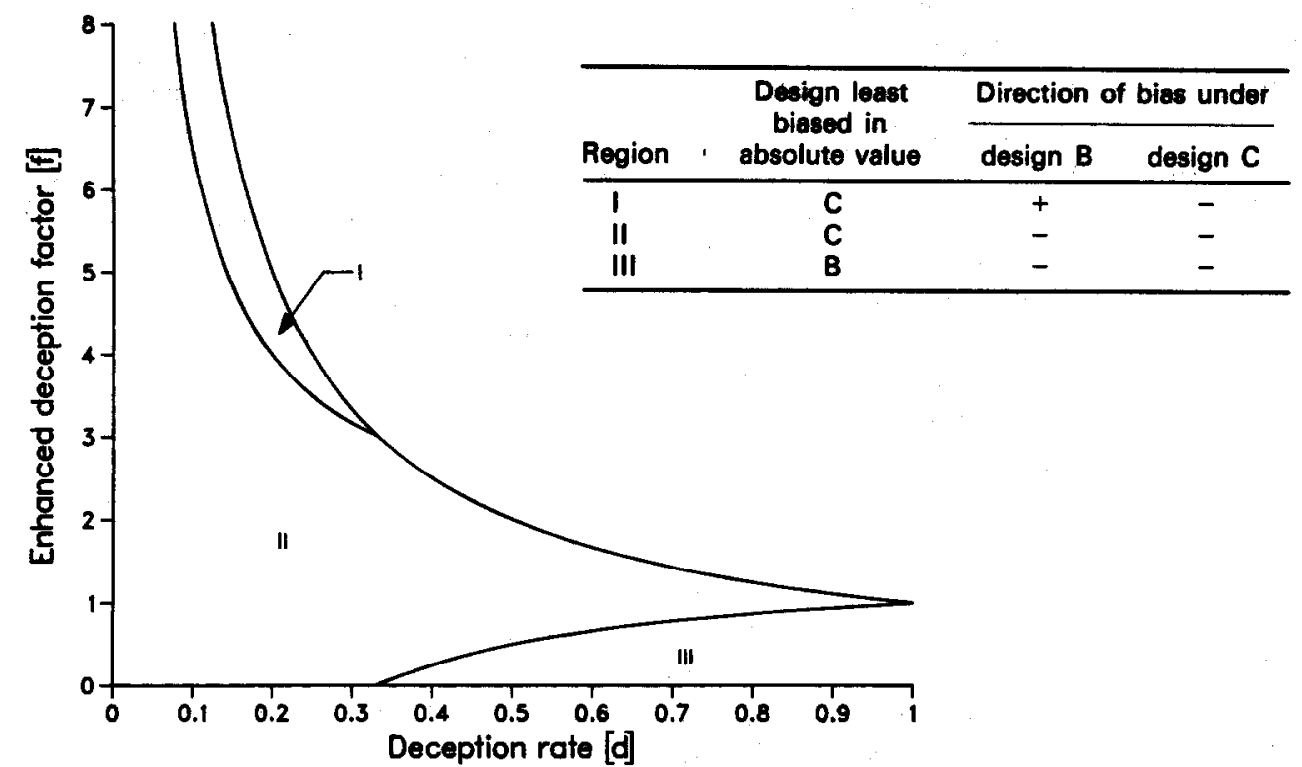

Fig. 3. Effect of deception rates on the expected relative bias. The figure shows three regions defining values for $d$ and $f$. Each region is unique with respect to the relative magnitudes (in absolute value) and direction of bias produced by two designs; outcomes classified solely by biochemical test results (design B) and by a combination of self-report among self-reported smokers and of biochemical test results among self-reported quitters (design C). The figure shows modeled results for $P_{1}=0.4, P_{0}=0.1, S_{i}=0.9$, and $S_{\mathrm{p}}=0.9$.

Each figure depicts three to five regions. Each region designates a range of values for $d$, the deception rate, and $f$, the factor by which deception is enhanced in the experimental group. Within a given region, Figs $1-3$ indicate which of the two strategies undergoing comparison is least biased (bias closest to zero). In theory, the deception rate $d$, a proportion, may assume values between 0 and 1 . The factor $f$, again in theory, may assume values between 0 and $d^{-1}$ (i.e. $0 \leqslant f \cdot d \leqslant 1$ ). For this reason, the upper right hand portions of Figs 1-3 (i.e. $f>d^{-1}$ ) are not defined by the model. Values for $f$ between 0 and 1 represent conditions when continued smokers in the experimental group are less likely to provide an incorrect self-report of nonsmoking. Figures 1-3 indicate, as well, the direction of bias produced by each strategy, when operating under the conditions specified by a given region. Trials with positive and negative bias overestimate and underestimate, respectively, the difference between the experimental and control treatments.

The curves, which divide Figs $1-3$ into regions, define values for $d$ and $f$ for which two strategies have equal bias. These curves derive from inequalities which employ expressions (1), (2), and (3) (Appendix B). These curves depend on the quit rates in the control $\left(P_{0}\right)$ and experimental $\left(P_{1}\right)$ groups and on the sensitivity $\left(S_{e}\right)$ and specificity $\left(S_{\mathrm{p}}\right)$ of the biochemical test for smoking. The specific curves in Figs 1-3 illustrate findings for $P_{0}=0.1, P_{1}=0.4, S_{\mathrm{e}}=0.9$, and $S_{\mathrm{p}}=0.9$.

In Fig. 1, the comparison between self-report (strategy A) and universal biochemical testing (strategy B) defines four regions. Strategy B produces the smallest relative bias when the deception rate $d$ and the enhanced deception factor $f$ fall into regions I or IV. Strategy A produces the smallest relative bias within regions II and III. Within region II, the relative bias is positive under designs which use selfreport, but negative under designs which use universal biochemical testing. In region II, the "preferred" strategy depends on whether it is regarded as more important to minimize bias or to guarantee against overestimation of the treatment effect (i.e. positive bias).

In Fig. 2, the comparison between self-report (strategy A) and selective biochemical testing (strategy C) defines five regions. Except within regions III and IV, the selective test use strategy produces the smallest relative bias. Within region I, the relative bias is positive whether the trial design uses self-report or selective bio- 
chemical testing. Within region III, use of selfreport results in a positive bias; selective test use produces a negative bias. Again, within region III, the "preferred" strategy depends on whether one wishes to minimize bias or to prevent positive bias.

In Fig. 3, the comparison between universal (strategy B) and selective (strategy C) testing strategies results in three regions. Except within region III, selective testing produces the trial with the smallest relative bias. Within region I, the relative bias is positive under strategy $B$ and negative under strategy $C$. Again, under the conditions specified within region I, the "preferred" strategy depends on whether one prefers to minimize bias or to prevent positive bias.

The curves which divide Figs 1-3 into regions and which, thereby, suggest the optimal testing strategy, depend on four parameters: $P_{0}, P_{1}, S_{\mathrm{e}}$, and $S_{\mathrm{p}}$. Changes in these parameters alter the sizes of the regions depicted. Shapes and general relationships among regions do not change. By inspection of equations (Appendix B) for these curves, we reach the following conclusions regarding the effects of changes in these parameters on choice of testing strategy with the smallest bias.

Increases in $P_{1}$ for constant $P_{0}$ (i.e. increases in treatment effectiveness) and increases in $P_{0}$ for constant $P_{1}-P_{0}$ (i.e. increases in control quit rates with treatment effectiveness left unchanged) have the same effects. Both increase $1-P_{0} / 1-P_{1}$ (the value for $f$ toward which all curves approach for high values of $d$ ) and $P_{1}-P_{0} / 1-P_{1}$ (a coefficient for $d^{-1}$ which determines the distance curves deviate from $\left.f=1-P_{0} / 1-P_{1}\right)$. Thus, in effect, the areas taken by regions II and III in Fig. 1 and by regions III and IV in Fig. 2 expand. That is, with higher quit rates, self-report produces less bias, for a wider range of possible values for $d$ and $f$, than biochemical testing.

Increases in test sensitivity $S_{e}$ have slightly more complex effects. Increases in $S_{\mathrm{e}}$, with one exception, favor strategies which make use of a biochemical test. Paradoxically, increases in $S_{\mathrm{e}}$ expand the area taken by region III in Fig. 2. That is, increases in $S_{\mathrm{e}}$ expand slightly the range of values for $f$ and $d$ over which the no testing strategy produces smaller relative biases than the strategy which tests self-reported quitters. In the former, but not the latter, instance, the relative bias is positive in direction.

Increases in test specificity $\left(S_{\mathrm{p}}\right)$, without exception, favor strategies which make use of a biochemical test. In addition, from equation (B2), we conclude that increases in $S_{\mathrm{p}}$ enlarge region $\mathrm{I}$ at the expense of region II, in Fig. 3. That is, a very specific biochemical test reduces the range of values for $f$ and $d$ over which the selective test use strategy produces a negative relative bias. Again, within region I (Fig. 3), universal biochemical testing is required to guarantee a trial not biased in favor of the experimental treatment.

\section{DISCUSSION}

The effects of misclassification on observational studies or clinical trials have received considerable attention [31, 32]. In the context of a clinical trial of treatments for smoking, misclassification of outcomes can arise from at least one of two sources, (1) inaccuracies in biochemical tests for smoking, used either to confirm self-report or to determine outcomes independently of self-report, and (2) misrepresentations, whether intentional or unintentional, of smoking status at the conclusion of study. We developed an algebraic model to examine the biases which result from these sources of misclassification [33].

Table 4 summarizes some principal findings from the model. In general, in the presence of equivalent treatments, a trial which uses selfreport or which tests self-reported quitters is positively biased; the magnitude of the bias is lower in the latter instance. In the absence of deceptive self-report, a trial which tests selfreported quitters or which tests all subjects is negatively biased; the magnitude of the bias is lower in the former instance. In the presence of equivalent deception rates in the control and experimental groups, a trial is always biased in a negative direction. Whether a biochemical test increases or decreases the magnitude of this bias depends on the precise relationships between characteristics of the test (i.e. sensitivity and specificity) and the deception rate.

The model also demonstrates that in the presence of a truly efficacious treatment, but with differential deception in control and experimental groups, increased sensitivity and specificity of a biochemical test, when used to validate the statements of self-reported quitters, have opposing effects on the bias. The model identifies the point beyond which, for a given test specificity, further improvements in test sensitivity result only in progressive bias in a negative direction (inequality 6 ). 
Finally, the model shows that the role biochemical tests should play in clinical trials of treatments for smoking depends on the quantitative relationships among deception rates and test characteristics. For one specific set of values for $P_{1}, P_{0}, S_{p}$, and $S_{e}$, Figs 1-3 display graphically which one of two paired designs, for given levels of deception in control or experimental groups, results in a trial least biased. Similar figures can be generated, for different values for $P_{1}, P_{0}, S_{\mathrm{p}}$, and $S_{\mathrm{e}}$, from equations provided in Appendix B. These figures emphasize that any judgement regarding the proper role of biochemical testing implies a quantitative statement regarding the magnitudes of deception in both the experimental and control groups. Unfortunately, our current state of knowledge permits only very imprecise estimates of the deception which occurs in actual practice (Tables 1-3).

We provide an example of application of these results during the design of an actual clinical trial of a treatment for smoking. Two biochemical tests were considered. The two tests differed in sensitivity, cost, and ease of administration. We used expression (3) to estimate the impact of each test on the amount of bias expected from a trial which used an imperfect test to confirm nonsmoking in self-reported quitters. For this purpose, we represented the expected effectiveness of the experimental intervention and the expected degree of deceptive self-report with the following parameters: $P_{0}=0.10, P_{1}=0.20, d=0.10$, and $f=2$. Under these conditions, the more expensive and accurate test $\left(S_{\mathrm{e}}=0.98, S_{\mathrm{p}}=0.98\right)$ would yield an observed treatment effect $1 \%$ smaller than the true treatment effect. The less expensive and less sensitive test $\left(S_{e}=0.90, S_{\mathrm{p}}=0.98\right)$ would yield an observed treatment effect $5 \%$ larger than the true treatment effect. We concluded that the less expensive test would satisfy the needs of a trial which aimed to limit the bias to $5 \%$.

In summary, the magnitude of deception, the determinants of deception, and the responsiveness of deception to changes in experimental protocol are uncertain. With this uncertainty, biochemical assessments of treatment outcomes from clinical trials must continue to play an important role, despite the risk of bias directed against observation of true treatment effects. Future trials should assess the value of biochemical tests in the context of expected deception rates in different treatment groups. More work is needed to improve the validity of self-reports of smoking and to understand deception among smokers as a phenomenon which not only complicates assessment of treatment programs for smoking, but may also relate with other factors which determine successful treatments for smoking.

\section{REFERENCES}

1. Fortmann SP, Rogers T. Vranian K, Haskell WL, Soloman DS, Farquhar JW. Indirect measures of cigarette use: Expired-air carbon monoxide vs plasma thiocynate. Prev Med 1984; 13: 127-135.

2. Pierce JP, Dwyer T, DiGiusto E, Carpenter T, Hannam C, Amin A, Yong C, Sarfaty G, Shaw J, Burke N. Cotinine validation of self-reported smoking in commercially run community surveys. J Curon Dis 1987; 40: 689-695.

3. Wald NJ, Idle $M$, Boneham J, Bailey A. Carbon monoxide in breath in relation to smoking and carboxyhemoglobin. Thorax 1981; 36: 366-369.

4. Cohen JD, Bartsch GE. A comparison between carboxyhemoglobin and serum thiocyanate as indicators of cigarette smoking. Am J Public Health 1980; 70: 284-286.

5. Robertson AS, Burge PS, Cockrill BL. A study of serum thiocyanate concentrations in office workers as a means of validating smoking histories and assessing passive exposure to cigarette smoke. Br J Indast Med 1987; 44: 351-354.

6. Jarvis MJ, Tunstall-Pedoe H, Feyerabend C, Vessey C, Saloojee $Y$. Comparison of tests used to distinguish smokers from nonsmokers. Am J Public Health 1987; 77: $1435-1438$.

7. Ronan G, Ruane P, Graham IM, Hickey N, Mulcahy R. The reliability of smoking history amongst survivors of myocardial infarction. Br J Addiet 1981; 76: 425-428.

8. Sillett RW, Wilson MB, Malcolm RE, Ball KP. Deception among smokers. Br Med J 1978; ii: 1185-1186.

9. Vessey CJ, Kirk CIC. Two automated methods for measuring plasma thiocyanate compared. Clin Chem 1985; 31: 270-274.

10. Wilcox RG, Hughes J, Roland J. Verification of smoking history in patients after infarction using urinary nicotine and cotinine measurement. Br Med $\mathbf{J}$ 1979; ii: 1026-1028.

11. Abrams DB, Follick MJ, Biener L, Carey KB, Hitti J. Saliva cotinine as a measure of smoking status in field settings. Am J Public Health 1987; 77: 846-848.

12. Colletti G, Supnick JA, Abueg FR. Assessment of the relationship between self-reported smoking rate and Ecolyzer measurement. Addict Behar 1982; 7: 183-188.

13. Glynn SM, Gruder CL, Jegerski JA. Effects of biochemical validation of self-reported cigarette smoking on treatment success and on misreporting abstinence. Health Prychol 1986; 5: 125-136.

14. Jamrozik K, Vossey M, Fowler G, Wald N, Parker G, VanVunakis H. Controlled trial of three different antismoking interventions in general practice. Br Med J 1984; 288: 1499-1503.

15. Lando HA. An objective check upon self-reported smoking levels: A preliminary report. Behav Ther 1975; 6: $547-549$.

16. Ohlin $\mathbf{P}$, Lundh B, Westling $H$. Carbon monoxide blood levels and reported cessation of smoking. Prychopharmacology 1976; 49: 263-265.

17. Russell MAH, Wilson C, Feyerabend C, Cole PV. Effect of nicotine chewing gum on smoking behavior and as an aid to cigarette withdrawal. Br Med J 1976; ii: $391-393$. 
18. Stookey GK, Katz BP, Olson BL, Drook CA, Cohen SL. Evaluation of biochemical validation measures in determination of smoking status. J Dent Res 1987; 66: 1597-1601.

19. Walker WB, Franzini LR. Low-risk aversive group treatments, physiological feedback, and booster sessions for smoking cessation. Behav Ther 1985; 16: 263-274.

20. Neaton JD, Broste S, Cohen L, Fishman EL, Kjelsberg M, Schoenberger J. The Multiple Risk Factor Intervention Trial (MRFIT): VII. A comparison of risk factor changes between the two study groups. Prev Med 1981; 10: 519-543.

21. Health and Public Policy Committee, American College of Physicians. Methods for stopping cigarette smoking. Ann Intern Med 1986; 105: 281-291.

22. Pechacek TF, Fox BH, Murray DM, Luepker RV Review of technique for measurement of smoking behavior. In: Matarazzo JD, Miller NE, Weiss SM et al., Eds. Behavioral Health: A Handbook of Health Enhancement and Disease Prevention. New York: John Wiley; 1984.

23. Bergers D, Junge B. Thiocyanate as an indicator of tobacco smoking. Prev Med 1979; 8: 351-357.

24. Vessey CJ, Saloojee Y, Cole PV, Russell MAH. Blood carboxyhemoglobin, plasma thiocyanate, and cigarette consumption: Implications for epidemiological studies in smokers. Br Med J 1982; 284: 1516-1518.

25. Paxton R, Bernacca G. Urinary nicotine concentration as a function of time since last cigarette: Implications for detecting faking in smoking clinic. Behav Ther 1979; 10: 523-528.

26. Jarvis MJ, Russell MAH, Feyerabend C, Eiser JR, Morgan M, Gammage P, Gray EM. Passive exposure to tobacco smoke: Saliva cotinine concentration in a representative population of school children. Br Med J 1985; 291: 927-929.

27. Stewart RD, Baretta ED, Platte LR, Stewart EB, Kalbfleisch JH, Van Yserloo B, Rimm AA. Carboxyhemoglobin levels in American blood donors. JAMA 1974; 229: 1187-1195.

28. Murray DM, O'Connell CM, Schmid LA, Perry CL. The validity of smoking self-report by adolescents: $A$ reexamination of the bogus pipeline procedure. Addict Behav 1987; 12: 7-15.

29. Petitti DB, Friedman GD, Kahn W. Accuracy of information on smoking habits provided on selfadministered questionnaire. Am J Public Health 1981; 71: 308-311.

30. Ockene JK, Pechacek TF, Vogt T, Svendsen K. Does switching from cigarettes to pipes or cigars reduce tobacco smoke exposure? Am J Public Health 1987; 77 : 1412-1416.

31. Flegal KM, Brownie C, Haas JD. The effects of exposure misclassification on estimates of relative risk. Am J Epidemiol 1986; 123: 736-751.

32. Sackett DI. Bias in analytic research. J Chron Dis 1979; 32: 51-63.

33. Feinstein AR, Horwitz RI. An algebraic analysis of biases due to exclusion, susceptibility, and protopathic prescription in case-control research. J Chron Dis 1981; 34: 393-403.

\section{APPENDIX A}

Consider clinical trial participants who distribute in the manner depicted in Table A1. By definition, the true treatment effect is

$$
\left(P_{11}+P_{12}\right)-\left(P_{21}+P_{22}\right)=P_{1}-P_{0} .
$$

The apparent treatment effect produced under conditions of deception is

$$
\begin{array}{r}
\left(P_{11}+P_{12}+P_{13}+P_{14}\right)-\left(P_{21}+P_{22}+P_{23}+P_{24}\right) \\
=P_{1}-P_{0}+\left(P_{13}+P_{14}\right)-\left(P_{23}+P_{24}\right) .
\end{array}
$$

But, by definition,

$$
d=\frac{P_{23}+P_{24}}{P_{23}+P_{24}+P_{25}+P_{26}}
$$

and

$$
f \cdot d=\frac{P_{13}+P_{14}}{P_{13}+P_{14}+P_{15}+P_{16}} .
$$

Substituting expressions (A2) and (A3) into (A1), the apparent treatment effect produced under conditions of deception then becomes

$$
\left(P_{1}-P_{0}\right)+d \cdot\left(f\left(1-P_{1}\right)-\left(1-P_{0}\right)\right),
$$

the absolute bias

$$
d \cdot\left(f\left(1-P_{1}\right)-\left(1-P_{0}\right)\right)
$$

and the relative bias

$$
\frac{d \cdot\left(f\left(1-P_{1}\right)-\left(1-P_{0}\right)\right)}{P_{1}-P_{0}} .
$$

The apparent treatment effect, when all participants are classified by a biochemical test for smoking, is

$$
\begin{aligned}
\left(P_{11}+P_{13}+P_{15}\right)-\left(P_{21}+P_{23}+P_{25}\right) & \\
= & \left(P_{11}+\left(1-P_{1}\right)-P_{14}-P_{16}\right) \\
& -\left(P_{21}+\left(1-P_{0}\right)-P_{24}-P_{26}\right) .
\end{aligned}
$$

By definition, the sensitivity $\left(S_{e}\right)$ of the biochemical test is

$$
\frac{P_{14}}{P_{13}+P_{14}}=\frac{P_{16}}{P_{15}+P_{16}}=\frac{P_{24}}{P_{23}+P_{24}}=\frac{P_{26}}{P_{25}+P_{26}}=S_{c}
$$

and the specificity $\left(S_{\mathrm{p}}\right)$

$$
\frac{P_{11}}{P_{11}+P_{12}}=\frac{P_{21}}{P_{21}+P_{22}}=S_{\mathrm{p}} \text {. }
$$

Expressing $P_{11}$ and $P_{21}$ in terms of $S_{\mathrm{p}}$ (expression A8) and $P_{14}, P_{16}, P_{24}$, and $P_{26}$ in terms of $S_{e}$ (expression A7), expression (A6), the apparent treatment effect, becomes

$$
\left(P_{1}-P_{0}\right)\left(S_{\mathrm{p}}+S_{\mathrm{e}}-1\right),
$$

the absolute bias

$$
\left(P_{1}-P_{0}\right)\left(S_{\mathrm{p}}+S_{\mathrm{e}}-1\right)-\left(P_{1}-P_{0}\right),
$$

and the relative bias

$$
S_{\mathrm{p}}+S_{\mathrm{e}}-2=-\left(\left(1-S_{\mathrm{p}}\right)+\left(1-S_{\mathrm{e}}\right)\right) .
$$

Finally, the apparent treatment effect, when self-reported quitters are classified by the biochemical test and when self-reported smokers are classified as smokers, is

$$
\begin{aligned}
\left(P_{11}\right. & \left.+P_{13}\right)-\left(P_{21}+P_{23}\right) \\
= & \left(P_{11}+\left(P_{13}+P_{14}\right)-P_{14}\right) \\
& -\left(P_{21}+\left(P_{23}+P_{24}\right)-P_{24}\right) .
\end{aligned}
$$

Expressing $\left(P_{13}+P_{14}\right)$ in terms of $f$ and $d$ (expression A3) $\left(P_{23}+P_{24}\right)$ in terms of $d$ (expression A2), $P_{11}$ and $P_{21}$ in terms of $S_{\mathrm{p}}$ (expression A8) and $P_{14}$ and $P_{24}$ in terms of $S_{\mathrm{c}}$ (expression A7), expression (A11), the apparent treatment effect, becomes

$$
\left(P_{1}-P_{0}\right) S_{\mathrm{p}}+d \cdot\left(f\left(1-P_{1}\right)-\left(1-P_{0}\right)\right)\left(1-S_{e}\right),
$$

the absolute bias

$$
d \cdot\left(f\left(1-P_{1}\right)-\left(1-P_{0}\right)\right)\left(1-S_{e}\right)-\left(P_{1}-P_{0}\right)\left(1-S_{p}\right),
$$

and the relative bias

$$
d \cdot\left(f\left(1-P_{1}\right)-\left(1-P_{0}\right)\right) \frac{1-S_{\mathrm{c}}}{P_{1}-P_{0}}-\left(1-S_{\mathrm{p}}\right) .
$$


Table Al. Cell probabilities for subjects enrolled in a clinical trial of treatments for smoking and classified according to true outcome, self-reported outcome, and biochemical test results

\begin{tabular}{|c|c|c|c|c|c|c|c|}
\hline & \multicolumn{2}{|c|}{ True quitter } & \multicolumn{4}{|c|}{ True smoker } & \multirow[b]{3}{*}{ Total } \\
\hline & \multirow[b]{2}{*}{ Neg } & \multirow[b]{2}{*}{ Pos } & \multicolumn{2}{|c|}{$\begin{array}{c}\text { Self-reported } \\
\text { quitter }\end{array}$} & \multicolumn{2}{|c|}{$\begin{array}{l}\text { Self-reported } \\
\text { smoker }\end{array}$} & \\
\hline & & & Neg & Pos & Neg & Pos & \\
\hline $\begin{array}{l}\text { Experimental group } \\
\text { Control group }\end{array}$ & $\begin{array}{l}P_{11} \\
P_{21}\end{array}$ & $\begin{array}{l}P_{12} \\
P_{22}\end{array}$ & $\begin{array}{l}P_{13} \\
P_{23}\end{array}$ & $\begin{array}{l}P_{14} \\
P_{24}\end{array}$ & $\begin{array}{l}P_{15} \\
P_{25}\end{array}$ & $\begin{array}{l}P_{16} \\
P_{26}\end{array}$ & $\begin{array}{l}1.00 \\
1.00\end{array}$ \\
\hline
\end{tabular}

Neg $=$ biochemical test indicates nonsmoking. Pos $=$ biochemical test indicates smoking.

\section{APPENDIX B}

The curves which separate Figs $1-3$ into regions derive from inequalities which make use of expressions (1), (2), and (3). The inequality

|expression A5 $|<|$ expression A10|,

$\left|\frac{d \cdot\left(f\left(1-P_{1}\right)-\left(1-P_{0}\right)\right)}{P_{1}-P_{0}}\right|<\left|-\left(\left(1-S_{\mathrm{p}}\right)+\left(1-S_{\mathrm{e}}\right)\right)\right|$

is used to derive the curves which separate Fig. 1 into regions:

region I from II,

$$
f=\frac{1-P_{0}}{1-P_{1}}+\left(\left(1-S_{\mathrm{p}}\right)+\left(1-S_{\mathrm{e}}\right)\right) \frac{P_{1}-P_{0}}{1-P_{1}} \frac{1}{d}
$$

region II from III,

$$
f=\frac{1-P_{0}}{1-P_{1}}, \text { and }
$$

region III from IV,

$$
f=\frac{1-P_{0}}{1-P_{1}}-\left(\left(1-S_{\mathrm{p}}\right)+\left(1-S_{\mathrm{e}}\right)\right) \frac{P_{1}-P_{0}}{1-P_{1}} \frac{1}{d}
$$

The inequality

|expression $\mathrm{A} 5 \mid$ < expression A13|,

$$
\begin{aligned}
& \left|\frac{d \cdot\left(f\left(1-P_{1}\right)-\left(1-P_{0}\right)\right)}{P_{1}-P_{0}}\right| \\
& <\left|d \cdot\left(f\left(1-P_{1}\right)-\left(1-P_{0}\right)\right) \frac{1-S_{e}}{P_{1}-P_{0}}-\left(1-S_{\mathrm{p}}\right)\right|
\end{aligned}
$$

is used to derive the curves which separate Fig. 2 into regions:

region I from II,

$$
f=\frac{1-P_{0}}{1-P_{1}}+\frac{1-S_{\mathrm{p}}}{1-S_{\mathrm{c}}} \frac{P_{1}-P_{0}}{1-P_{1}} \frac{1}{d}
$$

region II from III,

$$
f=\frac{1-P_{0}}{1-P_{1}}+\frac{1-S_{\mathrm{p}}}{1+\left(1-S_{\mathrm{e}}\right)} \frac{P_{1}-P_{0}}{1-P_{1}} \frac{1}{d}
$$

region III from IV, equation (BI) and

region IV from $V$,

$$
f=\frac{1-P_{0}}{1-P_{1}}-\frac{1-S_{\mathrm{p}}}{S_{\mathrm{e}}} \frac{P_{1}-P_{0}}{1-P_{1}} \frac{1}{d}
$$

The inequality

$$
\begin{aligned}
& \text { |expression } \mathrm{A} 10|<| \text { expression } \mathrm{A} 13 \mid \\
& \quad\left|-\left(\left(1-S_{\mathrm{p}}\right)+\left(1-S_{\mathrm{e}}\right)\right)\right| \\
& \quad<\left|d \cdot\left(f\left(1-P_{1}\right)-\left(1-P_{0}\right)\right) \frac{1-S_{\mathrm{e}}}{P_{1}-P_{0}}-\left(1-S_{\mathrm{p}}\right)\right|
\end{aligned}
$$

is used to derive the curves which separate Fig. 3 into regions:

region I from II, equation (B2), and region II from III,

$$
f=\frac{1-P_{0}}{1-P_{1}}-\frac{P_{1}-P_{0}}{1-P_{1}} \frac{1}{d}
$$

